\title{
EDITORIAL
}

\section{ALGUNAS RAZONES PARA CONSIDERAR LOS RIESGOS PSICOSOCIALES EN EL TRABAJO Y SUS CONSECUENCIAS EN LA SALUD PÚBLICA}

\author{
Pedro R Gil-Monte \\ Unidad de Investigación Psicosocial de la Conducta Organizacional (UNIPSICO). Universidad de Valencia.
}

Los factores psicosociales son las condiciones presentes en una situación laboral directamente relacionadas con la organización del trabajo, con el contenido del puesto, con la realización de la tarea e incluso con el entorno, que tienen la capacidad de afectar al desarrollo del trabajo y a la salud de las personas trabajadoras. Las expresiones organización del trabajo y factores organizativos se utilizan muchas veces de manera intercambiable con factores psicosociales para hacer referencia a las condiciones de trabajo que pueden conducir al estrés ${ }^{1}$.

Los factores psicosociales pueden ser favorables o desfavorables para el desarrollo de la actividad laboral y para la calidad de vida laboral del individuo. En el primer caso contribuyen positivamente al desarrollo personal de los individuos, mientras que cuando son desfavorables tienen consecuencias perjudiciales para su salud y para su bienestar. En este caso hablamos de factores de riesgo psicosocial o fuentes de estrés laboral y tienen el potencial de causar daño psicológico, físico, o social a los individuos. Son numerosos los estudios que han

Correspondencia:

Facultad de Psicología (Dep. Social)

Avda. Blasco Ibáñez, 21, 46010 Valencia

Tfno. 963864 564. Fax: 963864668

Correo electrónico: Pedro.Gil-Monte@uv.es concluido que los riesgos psicosociales son agentes capaces de deteriorar la salud de las personas durante el desempeño de su trabajo e incluso fuera de él ${ }^{2-4}$.

En la actualidad, los riesgos psicosociales en el trabajo son una de las principales causas de enfermedades y de accidentes laborales. Según la VI Encuesta de Condiciones de Trabajo en España elaborada por el Instituto Nacional de Seguridad e Higiene en el Trabajo ${ }^{5}$ el 70,9\% de los trabajadores encuestados señaló que estaba expuesto a riesgo de accidentes de trabajo -el porcentaje se eleva al $74,9 \%$ en el caso del personal sanitario-. El análisis de las causas de accidentes (v.g., riesgos de accidente por cortes y pinchazos) reveló que las principales causas de los riesgos se debían a distracciones, descuidos, despistes o falta de atención (45\%), trabajar muy rápido $(19,4 \%)$, y al cansancio o fatiga $(17,8 \%)$.

En el estudio se concluye que los trabajadores que se perciben expuestos a factores de riesgo psicosocial (v.g., sobrecarga de trabajo, exceso de carga mental, realización de tareas repetitivas y de muy corta duración) presentan porcentajes de respuesta significativamente mayores en sintomatología psicosomática (v.g., problemas de sueño, cansancio, dolores de cabeza, mareos, etc.) que los no expuestos. El $22,5 \%$ de los trabajadores encuestados 
consideran que el trabajo está afectando a su salud. Las dolencias que con más frecuencia atribuyen los encuestados al trabajo son: el dolor de espalda $(57,6 \%)$, el dolor de cuello $(28,1 \%)$, y el estrés $(27,9 \%)$. En relación con la presencia de otros síntomas, el cansancio, las alteraciones del sueño y las cefaleas inciden de forma importante sobre la población trabajadora, afectando al 12,3\%, $12 \%$ y $10,4 \%$, respectivamente.

Por otra parte, la Encuesta sobre Calidad de Vida Laboral realizada en 2007 por el Ministerio de Trabajo e Inmigración ${ }^{6}$ concluye que el $55,1 \%$ de las personas ocupadas manifiestan niveles medios o altos de estrés frente al 27,7\% que expresan niveles bajos o muy bajos. El nivel medio de estrés resultó igual en los varones y en las mujeres.

Estudios realizados en la Unión Europea (UE) han concluido que el estrés laboral es un problema importante y que supone un coste económico y social importante. El estrés laboral ocupa el cuarto lugar en la frecuencia de problemas de salud informados por los trabajadores de la UE. Un $22,3 \%$ de los trabajadores manifestaron que lo sufrían, según la Cuarta Encuesta Europea sobre Condiciones de Trabajo ${ }^{7}$. Además un 8,7\% respondieron tener problemas de sueño y un $7,8 \%$ problemas de ansiedad derivados del trabajo. Por sectores, la mayor frecuencia de problemas psicológicos la presentan los trabajadores de la educación, los de la sanidad, y los trabajadores de la administración pública en general.

Según recoge la Agencia Europea para la Seguridad y Salud en el Trabajo (http://osha. europa/en/topics/stress) el estrés es el segundo problema de salud relacionado con el trabajo informado con más frecuencia, afectando en 2005 al $22 \%$ de los trabajadores de la UE. Según estimaciones hechas en el 2002 el problema costaba a la
UE, formada entonces por 15 países, un mínimo de 20.000 millones de euros al año en concepto de tiempo perdido y gastos sanitarios.

En Estados Unidos, el estrés laboral constituye un problema similar al de la UE. Según un informe del National Institute for Occupational Safety and Health ${ }^{8}$, el porcentaje de trabajadores que informaron que su trabajo resultaba estresante estaba entre el $28 \%$ y el $40 \%$. En un estudio posterior se señala que los trabajadores afectados de ansiedad, estrés, o alteraciones neuróticas perdieron muchos más días de trabajo (25 días de promedio perdidos por trabajador) en el año 2001 que aquéllos que no presentaban este tipo de alteraciones (sólo 6 días como promedio perdidos por trabajador $)^{9}$.

Un informe publicado por la Agencia Europea para la Seguridad y la Salud en el Trabajo ${ }^{10}$ sobre riesgos psicosociales revela que los cambios técnicos u organizativos en el mundo laboral, junto con los cambios socioeconómicos, demográficos y políticos, incluido el fenómeno de la globalización, han originado riesgos psicosociales de carácter emergente que están teniendo consecuencias sobre la salud de la población debido a que pueden provocar un mayor estrés laboral y repercutir en la salud y la seguridad de los trabajadores. Estos riesgos han sido agrupados en cinco áreas: a) nuevas formas de contratación laboral, que se caracterizan por la aparición de contratos de trabajo más precarios y la subcontratación e inseguridad en el puesto de trabajo, b) envejecimiento de la población activa, que la hace más vulnerable a la carga mental y emocional, c) intensificación del trabajo, caracterizado por la necesidad de manejar cantidades de información cada vez mayores bajo una mayor presión en el ámbito laboral, d) fuertes exigencias emocionales en el trabajo, junto con un incremento del acoso psicológico y de la violencia, en especial en los sectores 
de la asistencia sanitaria y de los servicios en general, y e) desequilibrio y conflicto entre la vida laboral y personal, debido a la falta de ajuste entre las condiciones de trabajo y contratación y la vida privada de las personas.

Entre las consecuencias de los riesgos psicosociales, el síndrome de quemarse por el trabajo (SQT en adelante), también conocido como burnout, ha cobrado especial relevancia en las últimas décadas debido a un importante incremento de su prevalencia en el sector servicios, en especial en sanidad y educación ${ }^{11}$. Este fenómeno ha sido tipificado como enfermedad en algunos estudios ${ }^{12}$, y aunque no se reconoce como tal en el cuadro de enfermedades profesionales en el sistema de la Seguridad Social sí existen sentencias judiciales que le otorgan la condición de accidente de trabajo (véase el auto dictado por la Sala de lo Social del Tribunal Supremo de fecha 26 de octubre de 2000, Recurso Num: 4379/1999).

La prevalencia de esta patología, relacionada única y exclusivamente con el desempeño de la actividad laboral, varía de unos estudios a otros. En algunos estudios se han obtenido porcentajes del $30 \%^{13}$, aunque resultados más moderados sitúan su prevalencia en el $11,4 \%{ }^{14}$. Aplicando criterios más conservadores, en Holanda se estima que entre el $4 \%$ y el $7 \%$ de la población podrían sufrir niveles graves de $\mathrm{SQT}^{15}$. Porcentajes cercanos al $7 \%$ se han estimado en Suecia ${ }^{16}$. En Finlandia se estima que los casos graves de SQT de la población trabajadora se sitúan sobre el $2,4 \%{ }^{17}$. En estudios realizados en España se han obtenido porcentajes similares ${ }^{18}$.

Tras una revisión de la literatura Melamed et $\mathrm{al}^{3}$ concluyen que el SQT puede ser considerado un problema relevante para la salud pública y un tema de preocupación para los políticos responsables de la salud. La conclusión de estos autores no es desacertada. La dimensión del problema es merecedora de atención como problema de salud pública, pues es una patología que se desarrolla en mayor medida en los profesionales del sector servicios que desarrollan su actividad laboral hacia otras personas, y según la Encuesta de la Población Laboral Activa correspondiente al último trimestre de $2008^{19}$ un $70 \%$ de la población española ocupada trabaja en el sector servicios.

Trabajar en contacto con los clientes de la organización hace que los trabajadores estén expuestos con frecuencia a riesgos psicosociales como agresiones y conflictos interpersonales, sobrecarga de casos a atender, conflicto y ambigüedad de rol, percepción de inequidad en los intercambios sociales, falta de control sobre los resultados, etc. Estas condiciones de trabajo favorecen el desarrollo del SQT y el de otras patologías como la depresión.

El número de personas que sufren estrés por la exposición a los riesgos psicosociales en el trabajo va a ir en aumento, pues los cambios en el mundo laboral están incrementando el número de exigencias a los trabajadores, se pide mayor flexibilidad de destrezas y funciones, un mayor desequilibrio trabajo-familia, y más sobrecarga e intensificación del trabajo, a lo que se une el incremento de los contratos temporales, la subcontratación, y la falta de estabilidad en el empleo. Con relación a décadas pasadas, las estadísticas indican un incremento en los problemas de salud derivados de la exposición prolongada en el entorno laboral a los riesgos de carácter psicosocial.

Es cierto que existen algunos mitos sobre su prevalencia e incidencia, sustentados en parte por la información difundida desde la prensa, donde se pueden leer noticias como que entre el $71 \%$ y el $90 \%$ de los médicos de familia (La Voz de Galicia, 16 de marzo de $2004^{20}$ ), el $41,7 \%$ de los docentes (El País Comunidad Valenciana, 25 de mayo de 
$2002^{21}$ ), ó el $70 \%$ de los trabajadores sanitarios de Castilla-La Mancha (Europa Press, 19 de noviembre de 2002 ${ }^{22}$ ) están afectados de SQT. Pero, como se ha expuesto más arriba, estudios recientes han aplicado criterios más rigurosos y han corregido esas cifras a la baja, ofreciendo porcentajes más realistas. Aún así, las cifras siguen siendo lo suficientemente altas como para concluir que los riesgos psicosociales y las consecuencias que de ellos se derivan para la salud alcanzan niveles suficientemente elevados como para ser considerados una amenaza para la salud pública de la población.

De esta conclusión se derivan algunas recomendaciones: a) considerar los riesgos psicosociales como un problema en el sistema de salud pública español debería fomentar el desarrollo de sinergias entre las políticas desarrolladas en esta materia desde diferentes Ministerios, como el de Trabajo e Inmigración y el de de Sanidad y Consumo, b) esas sinergias deberían considerar la realización de estudios y encuestas de prevalencia que integren criterios e indicadores relevantes para la toma decisiones desde esos Ministerios, c) las normativas en materia de prevención de riesgos laborales deberían considerar la amenaza que para la salud pública de la población suponen los riesgos psicosociales en el trabajo y sus consecuencias, d) se deberían desarrollar campañas de prevención que fomentaran el desarrollo de planes de formación para los agentes sanitarios, de esta manera se contribuiría a erradicar diagnósticos erróneos o dilatados en el tiempo, y a mejorar algunos indicadores de la salud pública de la población española.

\section{BIBLIOGRAFÍA}

1. Sauter SL, Hurrell Jr JJ, Murphy LR, Levi L. Factores psicosociales y de organización. En: O.I.T. Enciclopedia de Salud y Seguridad en el Trabajo.
Madrid: Ministerio de Trabajo e Inmigración; 2001. p. 34.2-3. [citado 11 febrero 2009]. Disponible en: http://www.mtas.es/es/publica/enciclo/indice.htm

2. Dollard MF, LaMontagne AD, Caulfield N, Blewett V, Shaw A. Job stress in the Australian and international health and community services sector: a review of the literature. Int J Stress Manag. 2007; 14:417-45.

3. Melamed S, Shirom A, Toker S, Berliner S, Shapira I. Burnout and risk of cardiovascular disease: evidence, possible causal paths, and promising research directions. Psychol Bull. 2006; 132:327-53.

4. Quick JC, Tetrick LE, editores. Handbook of Occupational Health Psychology. Washington, DC: American Psychological Association; 2002.

5. INSHT. VI Encuesta de Condiciones de Trabajo. Madrid: MTAS; 2008.

6. MTIN. Encuesta de Calidad de Vida en el Trabajo. Madrid: Ministerio de Trabajo e Inmigración; 2007. [citado 11 febrero 2009]. Disponible en: http://www.mtin.es/estadisticas/ecvt/welcome.htm

7. Parent-Thirion A, Fernández E, Hurley J, Vermeylen G. Fourth European Working Conditions Survey. Luxembourg: Office for Official Publications of the European Communities; 2007.p.64 [citado 11 febrero 2009]. Disponible en: http://www.eurofound.europa.eu/pubdocs/2006/9 8/en/2/ef0698en.pdf

8. NIOSH. Stress... at work. Publication No. 99-101. Cincinnati, OH: NIOSH; 1999. [citado 11 febrero 2009]. Disponible en: http://www.cdc.gov/niosh/ pdfs/stress.pdf

9. NIOSH. Worker Health Chartbook 2004. Cincinnati, OH: NIOSH; 2004.p.34. [citado 11 febrero 2009]. Disponible en: http://www.cdc.gov/niosh/ docs/chartbook/

10. European Agency for Safety and Health at Work. Expert forecast on emerging psychosocial risks related to occupational safety and health. Luxembourg: Office for Official Publications of the European Communities; 2007.

11. Gil-Monte PR. El síndrome de quemarse por el trabajo (burnout). Una enfermedad laboral en la sociedad del bienestar. Madrid: Pirámide; 2005.

12. Masia J. El estrés laboral y el burnout en el mundo judicial: hacia un nuevo enfoque de la cuestión [citado 11 febrero 2009]. Disponible en: 
http://www.prevencionintegral.com/Estudios/Bur noutjudicial/default.htm

13. Albaladejo R, Villanueva R, Ortega P, Astasio P, Calle ME, Domínguez V. Síndrome de burnout en personal de enfermería de un hospital de Madrid. Rev Esp Salud Pública 2004; 79: 475-91.

14. Grau A, Flichtentrei D, Suñer R, Prats M, Braga F. El síndrome de burnout en profesionales sanitarios hispanoamericanos y españoles. Influencia de factores personales, profesionales y nacionales. Rev Esp Salud Pública 2009; 8: 215-230.

15. Schaufeli WB, Enzmann D. The burnout companion to study and practice: A critical analysis. Washington, DC: Taylor \& Francis; 1998.

16. Hallsten L. Burnout and wornout: Concepts and data from a national survey. En: Antoniou ASG, Cooper CL, editors. Research companion to organizational health psychology. Cheltenham, England: Edward Elgar; 2005. pp. 516-37.

17. Honkonen T, Ahola K, Pertovaara M, Isometä E, Kalimo R, Nykyri E, et al. The association between burnout and physical illness in the general population-results from the Finnish Health 2000 Study. J Psychosom Res 2006; 61: 59-66.
18. Gil-Monte PR, Carretero N, Roldán MD, NúñezRomán E. Prevalencia del síndrome de quemarse por el trabajo (burnout) en monitores de taller para personas con discapacidad. Rev Psicol Trab Organ 2005; 21:107-23.

19. Instituto Nacional de Estadística. Encuesta de Población Laboral Activa. Cuarto Trimestre de 2008 [citado 11 febrero 2009]. Disponible en: http://www.ine. es/daco/daco42/daco4211/epa0408.pdf

20. Redacción. El $80 \%$ de los médicos de familia han estado hartos de sus pacientes. La Voz de Galicia 2004 marzo 16 [citado 11 febrero 2009]. Disponible en: http://www.lavozdegalicia.es/hemeroteca/2004/03/16/2512936.shtml

21. Caballer N. UGT revela que el $41,7 \%$ de docentes está quemado y con problemas de salud. El PaísComunidad Valenciana 2002 mayo 25 [citado 11 febrero 2009]. Disponible en: http://www.elpais. com/articulo/Comunidad/Valenciana/UGT/revela/ 417/docentes/quemado/problemas/salud/elpepiespval/20020525elpval_33/Tes

22. Europa Press. El $70 \%$ de los trabajadores sanitarios de Castilla La Mancha sufren el síndrome de "burnout", según una encuesta de CCOO. Europa Press 2001 noviembre 18 [citado 11 febrero 2009]. Disponible en: http://www.psiquiatria.com/noticias/psicologia/psicologia_laboral/9526/ 
